\title{
Observation of an Emerging Charged Domain Wall at a Non-ferroelectric Heterointerface with Aberration-corrected STEM
}

\author{
Mengsha Li ${ }^{1}$, Changjian $\mathrm{Li}^{1}$, Chunhua Tang ${ }^{1}$, Stephen J. Pennycook ${ }^{1 *}$ \\ 1. Department of Materials Science and Engineering, National University of Singapore, Singapore, \\ Singapore. \\ * Corresponding author: steve.pennycook@nus.edu.sg
}

In ferroelectric materials, charged domain walls (CDWs) are particularly interesting as they break the polarization continuity. This discontinuity induces an accumulation of mobile carriers resulting in an enhanced conductivity [1]. For example, in $\mathrm{BaTiO}_{3}$ (BTO), electrical conductivity of a head-to-head domain wall is 6 orders of magnitude higher than in bulk [2]. Accounting for the narrow domain wall width (a few unit cells (ucs) only), it serves as a very confined conducting channel in an insulating matrix. Recent studies reveal that CDWs are not exclusive to ferroelectric materials, heterointerfaces between non-ferroelectric material are also capable of exhibiting CDWs. For example, at the $\mathrm{LaAlO}_{3}$ $(\mathrm{LAO}) / \mathrm{SrTiO}_{3}$ (STO) interfaces, the polar discontinuity drives polar distortions in either LAO or STO, as predicted by first principles calculations [3] and demonstrated by experiments [4,5]. Due to the advantageous properties of CDWs compared to the domains they separate, there is great promise in building novel devices based on CDWs, such as non-volatile ferroelectric domain wall memory devices [6]. Therefore, it has been said that "the wall is the device" [7]. To build a CDW-based low power device, the precise control of the CDWs, is a prerequisite.

Here, using atomic resolution scanning transmission electron microscopy (STEM) and electron energy loss spectroscopy (EELS), we show how to create and manipulate the position of the CDW in a nonferroelectric $\mathrm{LAO} / \mathrm{STO} / / \mathrm{NdGaO}_{3}$ heterostructure. Furthermore, with additional transport measurements, we show that CDWs, along with other factors, including oxygen octahedral rotation and interface intermixing, control the spatial distribution and density of the two-dimensional electron gas (2DEG) within the heterostructure.

As shown in Fig. 1a, the LAO/STO//NGO heterostructure consists two interfaces. CDWs and intermixing exhibit at the LAO/STO interface while octahedral rotation resides near the STO/NGO interface. From atomic resolution imaging, we discover a tail-to-tail CDW emerges at the LAO/STO interface when the STO thickness is larger than $20 \mathrm{ucs}$, accompanied by an increasing width of chemical intermixing (Fig. 1d). Furthermore, the oxygen octahedral rotation (OOR) from the NGO substrate affects the Ti-O-Ti bond angle within the STO layer for 4 ucs (Fig. 1f). Both intermixing and distorted Ti-O-Ti bonds induce carrier localization in the 2DEG. As a result, when we change the STO thickness, we effectively tune the spatial distribution of mobile carriers, which is revealed by EELS Ti valence mapping. Specifically, when the STO layer is thick enough ( $\geq 6 \mathrm{ucs}$ ), a conducting channel forms, which is free from carrier localization due to either intermixing or OOR. The $\mathrm{Ti}^{3+}$ concentration within the conducting channel (Fig. 1g) correlates accurately with transport measurements. In summary, CDW creation, and its relationship with intermixing, directly control the 2DEG conducting channels, thus offering novel approaches for CDW based functional devices [8]. 
References

[1] M. P. Campbell et al., Nat. Commun. 7 (2016), p. 13764.

[2] Tomas Sluka et al., Nat. Commun. 4 (2013), p. 1808.

[3] Rossitza Pentcheba, Wattrn E. Pickett, Phys. Rev. Lett. 102 (2009) p. 107602.

[4] Frank Schoofs et al., J. Phys. Condens. 25 (2013) p. 175005.

[5] C. Cantoni et al., Adv. Mater. 24 (2012) p. 3952.

[6] Pankaj Sharma et al., Sci. Adv. 3 (2017) p. 1700512.

[7] G. Catalan et al., Rev. Mod. Phys. 84 (2012) p. 119.

[8] The authors acknowledge financial support from the Lee Kuan Yew Postdoctoral Fellowship through the Singapore Ministry of Education Academic Research Fund Tier 1 (R-284-000-158-114) and the Ministry of Education, Singapore under its Tier 2 Grant (Grant No. MOE2017-T2-1-129).
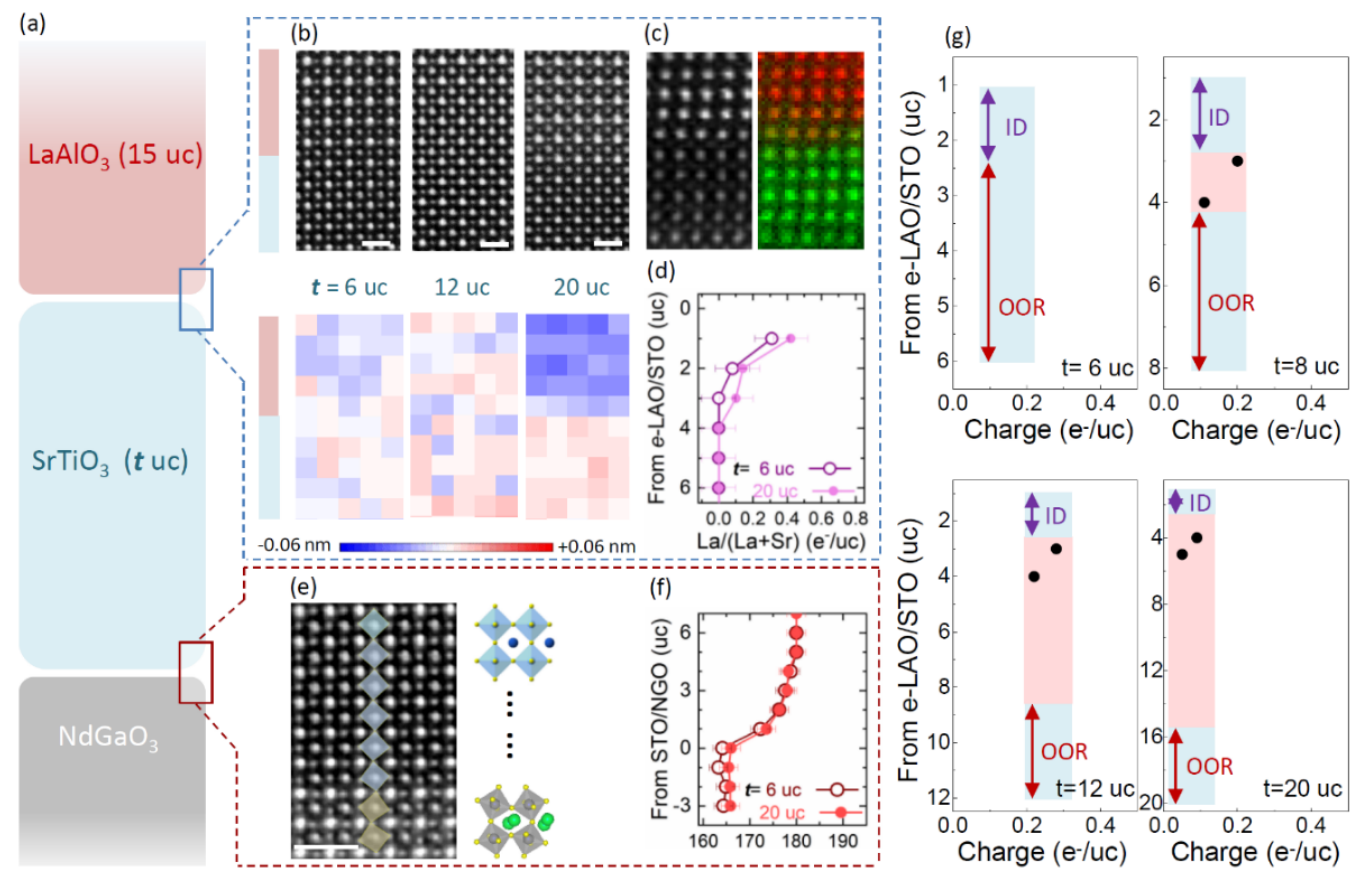

Figure 1. (a) Schematic diagram of the LAO/STO//NGO heterostructure with 15 uc LAO layer and t uc STO layer. (b) Inverted ABF-STEM images after radial difference filtering with corresponding relative displacement (oxygen atoms relative to the B-cations, $\delta_{z}$ ) maps of the LAO/STO interfaces, with 6, 12 and 20 ucs of STO (scale bars: $0.5 \mathrm{~nm}$ ). The negative (positive) sign, shown in blue (red), represents the polarization pointing away from the LAO/STO interface in the LAO (STO) side. (c) Simultaneous ADF image and La+Sr EELS map at the LAO/6-STO interface to show interdiffusion of La into the STO side. (d) The cation ID at the LAO/6-STO and LAO/20-STO interfaces. (e) Inverted ABF-STEM image of the 6-STO/NGO interface after radial difference filtering along the [1ㅜㅣ] NGO zone axis (scale bar, $1 \mathrm{~nm}$ ).

(f) Calculated M-O-M (M=Ti, Ga) bond angle $(\theta)$ as a function of distance to the STO/NGO interface in the 6 and 20 uc samples. (g) The $\mathrm{Ti}^{3+}$ concentration as a function of distance to LAO/STO interface in the heterostructures with 6, 8, 12 and 20 uc STO. The purple and red arrows represent the regions affected by intermixing and OOR, respectively. The conducting channel is denoted in pink. 